\title{
Jun, Uwe, und Oskar Niedermayer (Hrsg.) (2020): Die Parteien nach der Bundestagswahl 2017. Aktuelle Entwicklungen des Parteienwettbewerbs in Deutschland Wiesbaden: Springer VS. 252 Seiten. 49,99€
}

\author{
Kristina Weissenbach (D)
}

Angenommen: 31. März 2021 / Online publiziert: 28. April 2021

(C) Der/die Autor(en) 2021

Wie ist die Lage der politischen Parteien und des Parteiensystems in Deutschland nach der Bundestagswahl 2017? Zwar stellen Uwe Jun und Oskar Niedermayer, die Herausgeber des Bandes „Die Parteien nach der Bundestagswahl 2017“, diese Frage eingangs nicht selbst - auf 252 Seiten wird sie in acht Aufsätzen jedoch vielseitig diskutiert.

Eingeleitet wird der Sammelband - ebenso wie seine seit der Bundestagswahl 1998 erscheinenden Vorgängerbände - mit einem Aufsatz von Oskar Niedermayer zur Struktur und Entwicklung des deutschen Parteiensystems (S. 1-42). Darauf folgen je circa 30-seitige Einzelfallanalysen der sieben im Bundestag vertretenen Parteien, absteigend sortiert nach ihrem Zweitstimmenanteil bei der Wahl. Diese sind, mit geringfügigen Abweichungen und je eigenen thematischen Schwerpunktsetzungen, gleich strukturiert: Ein erster Abschnitt erzählt die Ausgangslage der Partei vor der Bundestagswahl 2017, ein zweiter befasst sich mit dem Wahlkampf der Partei, in einem dritten Schritt besprechen die Autoren Wahlergebnisse und -verhalten sowie Wählerdemografie, zuletzt werden innerparteiliche Entwicklungen und Zukunftsoptionen nach der Bundestagswahl 2017 dargelegt.

Das Eingangskapitel gibt den Leserinnen und Lesern lehrbuchartig einen Überblick über klassische Ansätze der vergleichenden Parteiensystemforschung zur Typisierung von Parteiensystemen. Dabei bezieht der Beitrag sowohl statische Struktureigenschaften wie Fragmentierung oder Asymmetrie mit ein wie auch die Prozessperspektive des Wandels von Parteiensystemen (Volatilität, Polarisierung, Segmentierung). Andere elektorale und gouvernementale Aspekte wie Legitimität, Regierungsstabilität oder Institutionalisierung werden nicht adressiert. Nach umfassender

K. Weissenbach $(\bowtie)$

Universität Duisburg-Essen, Duisburg, Deutschland

E-Mail: kristina.weisenbach@uni-due.de

University of Washington, Seattle, USA 
Analyse der Strukturveränderungen des deutschen Parteiensystems von 1949 bis 2017, mit Fokus auf Positionsveränderungen der Parteien entlang der wirtschaftsund gesellschaftspolitischen Konfliktlinie zwischen 2013 und 2017, kommt Niedermayer zu dem Schluss, dass sich ,das deutsche Parteiensystem, das von Anfang an und über sechs Jahrzehnte hinweg ein System mit Zweiparteiendominanz war, zu einem pluralistischen System an der Grenze zum hochfragmentierten System entwickelt" (S. 40).

Gewinnbringend für die Strukturierung der Fallstudien wäre es nun gewesen, wenn dieser theoretisch-methodische Rahmen stärker Eingang in die Einzelfallanalysen gefunden hätte. Mit Ausnahme der Kapitel der Herausgeber selbst folgen die Analysen jedoch je eigenen Zugängen.

So fragt Uwe Jun in seinem Beitrag (S. 71-104) danach, wie sich die SPD im Parteienwettbewerb positioniert und welche Rolle sie im Regierungssystem entwickelt. Er behandelt vorwiegend die Gründe für ihren elektoralen Niedergang und diskutiert den Entfremdungsprozess zwischen der Partei und ,,ihren vormaligen Traditionswählern aus den sozial geringer privilegierten Gruppen“ (S. 76). Erklärungskräftig erscheint dabei der Vergleich der SPD zur internationalen Sozialdemokratie und der Befund, dass die SPD hinsichtlich „Policy-Gaps“ (Gagné und Hilmer 2019) „Zwischen ihrer traditionellen Wählerschaft unter sozialdemokratischen Parteien am schlechtesten ab[schneidet]“ (S. 78) - vor allem im Bereich der soziokulturellen Wettbewerbsdimension und viel geringer in der sozioökonomischen Dimension.

Niedermayers Kapitel zur AfD (S. 105-132) gibt interessante Einblicke in eine Lebensphase junger Parteien, die unmittelbar mit der Parteiensystementwicklung zusammenhängt: Das Überschreiten der Repräsentationsschwelle, der Eintritt in das (nationale) Parlament. Er schließt dabei jedoch weniger an den Diskurs um die Rolle neuer Parteien in etablierten Parteiensystemen an, sondern analysiert detailgenau den Wandel der programmatischen Positionierung und personelle Entwicklungen sowie Fragen der Wählerwanderung und der parteipolitischen Zusammensetzung der AfDWählerschaft.

Die weiteren Texte spiegeln erkennbar die - nicht minder interessanten - individuellen Forschungsschwerpunkte und konzeptionellen Herangehensweisen der Autoren. So behandeln die Kapitel über CDU und Bündnis 90/Die Grünen stärker kommunikative, auf Wahlkampf und Wahlkampfstrategie bezogene Aspekte.

Torsten Oppellands aufschlussreiche Auseinandersetzung (S. 43-70) mit den Mitteln des CDU-Wahlkampfes greift die in der vergleichenden Parteienforschung diskutierte Frage auf ,wie etablierte Mainstream-Parteien auf neu ins Parteiensystem eintretende rechtspopulistische Parteien reagieren können“ (S. 47) und wird mit zwei strategischen Elementen der CDU-Wahlkampfführung beantwortet: Ein ,Formelkompromiss mit der CSU, der den Ausschluss jeglicher Kooperation mit der AfD mit beinhaltete [sowie] eine Feel-Good-Strategie für den Wahlkampf“" (S. 46). Der entscheidende wahlkampfstrategische Schritt, so Oppelland, war jedoch ,die richtige Reaktion auf den Schulz-Hype“ (S. 46) - ausharren und Ruhe bewahren, ,auch wenn man in der Partei nervös zu werden begann“ (S. 49).

In seiner detailgenauen Analyse der Grünen-Wahlkampfstrategie kontrastiert Lothar Probst (S. 187-220) das dürftige Wahlergebnis von 8,9 Prozent mit der höhenflugartigen Entwicklung der Partei in den darauffolgenden Jahren. Gewinnbringend 
ist der Blick auf die Grünen-Regierungsbeteiligungen auf Landesebene und die damit einhergehende Professionalisierung, Machtverschiebungen und informelle Aspekte des Abstimmungsprozesses mit den Bundesgrünen wie die neu geschaffenen Treffen der „G-Koordination“ (S. 193). Diese sollten „die parteiinterne Koordination bezüglich des Abstimmungsverhaltens im Bundesrat effektiver gestalten, andererseits aber sollten dadurch auch die Positionen zwischen Vertretern der Landesregierungen sowie der Partei- und Fraktionsspitze soweit wie möglich harmonisiert werden“" (S. 194).

Die Analysen der FDP, der Linken sowie der CSU fokussieren parteiorganisatorische, programmatische und personelle Aspekte.

So gibt der Beitrag von Benjamin Höhne und Uwe Jun (S. 133-158) einen profunden und empirisch reichhaltigen Einblick in die organisatorische und programmatische Entwicklung der FDP sowie die ideologischen Distanzen zu den anderen Bundestagsparteien. Unter Einbezug des sorgfältig aufgearbeiteten Forschungsstands und basierend auf Daten aktueller Umfrageforschung rekonstruiert der Aufsatz anschaulich die Lebenszyklen der FDP, den externen Druck sowie den Organisationsreformprozess der Partei seit dem Ausscheiden aus dem Bundestag 2013.

In seinem gut strukturierten Kapitel über die Linke (S. 159-186) konfrontiert Hendrik Träger die Partei nach der Bundestagswahl 2017 mit zentralen Befunden der Analysen von Neugebauer und Stöss (1999, 2003, 2007, 2015) aus den vorangegangenen Bänden: „Wurde die Linke auch im September 2017 vorrangig als Ausdruck des Protests gewählt?“ (S. 160) - laut Träger, nein. „Ist die Linke weiterhin hauptsächlich eine Fraktionspartei?" (S. 161) - ja. Ist die Linke auf dem Höhepunkt ihrer Ausdehnung? Träger bestätigt hier das Ergebnis von Neugebauer und Stöss, dass die „Westausdehnung“ (S. 161) faktisch nicht erfolgreich war.

Der Sammelband schließt mit Michael Weigls Auseinandersetzung mit der Lage der CSU (S. 221-251), die er als ,weit mehr als nur eine Wahlniederlage“ (S. 221) beschreibt. Für ihn markiert das Bundestagswahlergebnis ,,[... ein Erdbeben, das die inzwischen baufällige Substanz des einst stolzen Hauses CSU vor Augen“ (S. 221) führt. Im Weiteren behandelt der Beitrag programmatische, organisationale sowie personelle Entscheidungen der CSU nach der Bundestagswahl 2017 und stellt dabei die Frage danach, ,wie viel Konservativismus einer modernisierten Union noch zu Gesicht“" (S. 224) steht, in das Zentrum.

Der große Mehrwert der seit der Bundestagswahl 1998 erscheinenden Bände zur Entwicklung des Parteienwettbewerbs in Deutschland ist die Möglichkeit des Vergleichs im Längsschnitt über Zeit. Eine querschnittsvergleichende Position einzunehmen, Schlussfolgerungen im Vergleich der Parteien zu ziehen und vielfältige Perspektiven kennenzulernen wird den Leserinnen und Lesern jedoch dadurch erschwert, dass der Herausgeberband keine konzeptionelle Rahmung vorweist, auch keine Hinführung zu den Einzelfallanalysen oder abschließende vergleichende Einordnung und nur punktuell Bezüge zur internationalen Parteienforschung herstellt.

Die umfassende Rekonstruktion der (partei-)politischen Lage Deutschlands nach der Bundestagswahl 2017 ist die Stärke des Bandes. Die sorgfältig aufgearbeiteten Einzelfallanalysen lassen Daten der amtlichen Wahlstatistik und Umfrageforschung genauso sprechen wie die handelnden Parteiakteure selbst oder Partei- und Wahlprogramme. Auch die Rezeption von Wahlkampf und Parteienentwicklung seitens 
der (sozialen) Medien findet Eingang in einige der Analysen. Der interessante und gut lesbare Band setzt damit die Situation der Parteien in Deutschland nach der Bundestagswahl 2017 in den Kontext aktueller inner- und außerparteilicher Diskussionen und Streitfragen. Er bereichert unser Verständnis zur objektiven, internen und externen Entwicklung der deutschen Parteien und sei daher allen empfohlen, die die Wahl 2017 nachbearbeiten und sich umfassend auf das Wahljahr 2021 vorbereiten möchten.

Funding Open Access funding enabled and organized by Projekt DEAL.

Open Access Dieser Artikel wird unter der Creative Commons Namensnennung 4.0 International Lizenz veröffentlicht, welche die Nutzung, Vervielfältigung, Bearbeitung, Verbreitung und Wiedergabe in jeglichem Medium und Format erlaubt, sofern Sie den/die ursprünglichen Autor(en) und die Quelle ordnungsgemäß nennen, einen Link zur Creative Commons Lizenz beifügen und angeben, ob Änderungen vorgenommen wurden.

Die in diesem Artikel enthaltenen Bilder und sonstiges Drittmaterial unterliegen ebenfalls der genannten Creative Commons Lizenz, sofern sich aus der Abbildungslegende nichts anderes ergibt. Sofern das betreffende Material nicht unter der genannten Creative Commons Lizenz steht und die betreffende Handlung nicht nach gesetzlichen Vorschriften erlaubt ist, ist für die oben aufgeführten Weiterverwendungen des Materials die Einwilligung des jeweiligen Rechteinhabers einzuholen.

Weitere Details zur Lizenz entnehmen Sie bitte der Lizenzinformation auf http://creativecommons.org/ licenses/by/4.0/deed.de. 